\title{
Postoperative radiotherapy for invasive micropapillary carcinoma of the breast: an analysis of Surveillance, Epidemiology, and End Results database
}

\author{
San-Gang Wu' ${ }^{1, *}$ \\ Wen-Wen Zhang',* \\ Jia-Yuan Sun ${ }^{2}$ \\ Feng-Yan $\mathrm{Li}^{2}$ \\ Yong-Xiong Chen ${ }^{3}$ \\ Zhen-Yu $\mathrm{He}^{2}$ \\ 'Department of Radiation Oncology, \\ Xiamen Cancer Hospital, The First \\ Affiliated Hospital of Xiamen \\ University, Xiamen, ${ }^{2}$ Department \\ of Radiation Oncology, Sun Yat-sen \\ University Cancer Center, State Key \\ Laboratory of Oncology in South \\ China, Collaborative Innovation \\ Center of Cancer Medicine, \\ Guangzhou, ${ }^{3}$ Eye Institute of Xiamen \\ University, Fujian Provincial Key \\ Laboratory of Ophthalmology and \\ Visual Science, Medical College, \\ Xiamen University, Xiamen, People's \\ Republic of China \\ *These authors contributed equally to \\ this work
}

Correspondence: Yong-Xiong Chen Eye Institute of Xiamen University, Fujian Provincial Key Laboratory of Ophthalmology and Visual Science, Medical College, Xiamen University, Xiang'an South Road, Xiang'an District, Xiamen 36II02, People's Republic of China

Tel +865922183761

Fax +865922186786

Email yxchen 1962@xmu.edu.cn

Zhen-Yu He

Department of Radiation Oncology, Sun Yat-sen University Cancer Center, State Key Laboratory of Oncology in South China, Collaborative Innovation Center of Cancer Medicine, 65I Dongfeng

Road East, Guangzhou 5I 0060, People's

Republic of China

Tel +86 2087343543

Fax+86 2087343392

Email hezhy@sysucc.org.cn
This article was published in the following Dove Press journal:

Cancer Management and Research

3 October 2017

Number of times this article has been viewed

Introduction: Invasive micropapillary carcinoma (IMPC) of the breast poses a high risk of locoregional recurrence, and postoperative radiotherapy (PORT) may be beneficial in IMPC. Hence, we determined the clinical value of PORT in IMPC patients.

Patients and methods: We assessed clinicopathological factors extracted from the Surveillance, Epidemiology, and End Results database (2004-2013). Univariate and multivariate Cox proportional hazards regressions were performed to assess the independent prognostic factors on breast cancer-specific survival (BCSS) and overall survival (OS).

Results: Of the 881 study patients, 444 (50.4\%) and 437 (49.6\%) underwent breast-conserving surgery (BCS) and mastectomy (MAST), respectively, of whom 357 (80.4\%) and $153(35.0 \%)$ underwent PORT, respectively. Patients with young age, large tumor size, or advanced nodal stage were more likely to undergo MAST and PORT compared with MAST alone. Patients with progesterone receptor-positive disease were more likely to receive BCS and PORT compared with BCS alone. The 5-year BCSS and OS were $95.7 \%$ and $90.9 \%$, respectively. On multivariate analyses, tumor size, histological grade, and estrogen receptor status were independent predictors of BCSS and OS. The types of surgical procedures (MAST vs. BCS) were not an independent predictor of survival outcomes. Patients who underwent MAST with or without PORT had similar BCSS and OS in the multivariate analyses. Those who underwent BCS plus PORT did not have better BCSS and OS than those who underwent BCS alone. In the low-, intermediate-, and high-risk groups, PORT was not associated with better BCSS and OS than non-PORT groups in patients who received BCS or MAST.

Conclusion: IMPC has favorable BCSS and OS. Regardless of the types of surgical procedures (MAST or BCS), PORT groups were not inferior to non-PORT groups on BCSS and OS.

Keywords: invasive micropapillary carcinoma, SEER, radiotherapy, survival

\section{Introduction}

Invasive micropapillary carcinoma (IMPC) is a rare histological subtype of breast carcinoma, accounting for 3\%-6\% of all invasive breast cancers. ${ }^{1}$ IMPC was first described in 1993, and the World Health Organization classified IMPC as an independent breast tumor in 2003. ${ }^{1,2}$ It is generally accepted that IMPC is associated with a higher probability of lymphovascular invasion (LVI) and regional lymph node metastasis $(66 \%-90 \%)$ than invasive ductal carcinoma (IDC). ${ }^{3-10}$ Abnormal expression of several markers, including MUC1, N-cadherin, E-cadherin, and CD44, may be related to the highly aggressive tumor biology, resulting in lymph node metastases, high recurrence 
rates, and short disease-free survival. ${ }^{11-21}$ However, whether IMPC histology is an independent prognostic factor in breast cancer remains controversial. Several studies have reported that there is no significant difference in outcomes between IMPC and IDC patients matched for lymph node status. ${ }^{22-24}$ Two population-based studies that contributed to the Surveillance, Epidemiology, and End Results (SEER) database have also confirmed that survival outcomes are similar for the IMPC and IDC subtypes. ${ }^{25,26}$

Postoperative radiotherapy (PORT) is an important adjuvant therapy in breast cancer patients after breastconserving surgery (BCS) or in high-risk patients after mastectomy (MAST). ${ }^{27,28}$ Several studies have found that the locoregional recurrence (LRR) rate of IMPC is higher than that of IDC, ${ }^{10,29}$ possibly because unlike IDC, IMPC is associated with several high risk factors for LRR, including LVI and lymph node metastasis. Therefore, PORT may play an important role in IMPC. However, the clinical value of PORT in patients with IMPC remains unclear. In this study, we used the SEER database to determine the clinical value of PORT in patients with IMPC.

\section{Patients and methods}

\section{Patients}

The study population consisted of patients with pathologically proven IMPC of the breast treated between 2004 and 2013 , according to the current SEER program. ${ }^{30}$ The inclusion criteria for this study were as follows: 1) women with localized or regional IMPC of the breast; 2) IMPC as the primary cancer diagnosis; 3) treated with local surgery including MAST and BCS; 4) availability of data on whether postoperative beam radiation was performed; and 5) availability of data on ethnicity, tumor size, histological grade, nodal stage, and estrogen receptor (ER) and progesterone receptor (PR) statuses. This study was approved by the ethics committee of The First Affiliated Hospital of Xiamen University and Sun Yat-sen University Cancer Center.

\section{Clinicopathological factors}

The following clinicopathological factors were extracted from the SEER database: age at diagnosis, ethnicity, tumor size ( $\mathrm{T}$ stage), histological grade, lymph node staging $(\mathrm{N}$ stage), surgical procedures, and hormone receptor status. Lymph node stage was based on the number of metastatic lymph nodes, according to the current Union for International Cancer Control/American Joint Committee on Cancer staging system.

\section{Statistical analysis}

Statistical analyses were performed using the SPSS statistical software package (version 21.0; IBM Corporation, Armonk, NY, USA). The $\chi^{2}$ test and Fisher's exact probability test were used to evaluate differences between qualitative variables. Breast cancer-specific survival (BCSS) and overall survival (OS) rates were estimated by the Kaplan-Meier method and compared with the log-rank test. Univariate and multivariate analyses were performed to investigate the risk factors for BCSS and OS by using the Cox proportional hazards model. For all statistical analyses, a $p$ value of $<0.05$ was considered as significant.

\section{Results}

\section{Patient characteristics and treatment}

In total, 881 patients with IMPC of the breast were included in this study. The characteristics of the patients are listed in Table 1. The median age was 59 years (range, 25-95 years). Of the 881 patients, 511 (58.0\%) had T1 stage disease. The median tumor size was $18 \mathrm{~mm}$. A total of $344(39.0 \%)$ patients had poorly differentiated or undifferentiated tumors, and 462 (52.4\%) patients had node-positive disease. ER and PR positivity was observed in $90.0 \%$ and $77.0 \%$ of patients, respectively.

A total of 444 (50.4\%) and 437 (49.6\%) patients underwent BCS and MAST, respectively. In each group, 357 $(80.4 \%)$ and $153(35.0 \%)$ patients received PORT, respectively. Patients with younger age ( $\leq 49$ years), larger tumors (T2-3 stages), and advanced nodal stage (N2-3 stages) were more likely to have received MAST and PORT compared to patients who received MAST alone. In BCS groups, patients with PR-positive disease were more likely to receive BCS with PORT. There were no significant differences in age, ethnicity, tumor size, tumor grade, nodal stage, and ER status between patients who received BCS alone and those who received BCS with PORT.

\section{Factors influencing patient survival}

The median follow-up was 39 months (range, 0-119 months). A total of 64 patients died, including 31 patients who died of breast cancer-related disease. The 5-year BCSS and OS rates were $95.7 \%$ and $90.9 \%$, respectively. Univariate analyses revealed that age, tumor size, histological grade, nodal stage, ER status, and PR status were prognostic factors for BCSS and OS (Table 2). Patients who had received MAST had significantly worse BCSS and OS than those who had received $\mathrm{BCS}$. 
Table I Clinicopathological characteristics of the study patients

\begin{tabular}{|c|c|c|c|c|c|c|c|c|}
\hline Characteristics & $\mathbf{n}$ & BCS & BCS + RT & MAST & MAST + RT & $p^{a}$ & $p^{b}$ & $p^{c}$ \\
\hline \multicolumn{9}{|l|}{ Age (years) } \\
\hline$\leq 49$ & 204 & $14(15.1)$ & $55(15.4)$ & $74(26.1)$ & $61(39.9)$ & $<0.001$ & 0.237 & 0.011 \\
\hline $50-69$ & 477 & $44(50.6)$ & $213(59.7)$ & 152 (53.5) & $68(44.4)$ & & & \\
\hline$\geq 70$ & 200 & $29(33.3)$ & 89 (24.9) & $58(20.4)$ & $24(15.7)$ & & & \\
\hline \multicolumn{9}{|l|}{ Ethnicity } \\
\hline White & 682 & $64(73.6)$ & 278 (77.9) & $226(79.6)$ & II 4 (74.5) & 0.858 & 0.637 & 0.457 \\
\hline Black & 107 & $13(14.9)$ & $4 \mid(11.5)$ & $31(10.9)$ & $22(14.4)$ & & & \\
\hline Others & 92 & $10(11.5)$ & $38(10.6)$ & $27(9.5)$ & $17(11.1)$ & & & \\
\hline \multicolumn{9}{|l|}{ Tumor size } \\
\hline $\mathrm{TI}$ & 511 & 65 (74.7) & $259(72.5)$ & I53 (53.9) & 34 (22.2) & $<0.001$ & 0.916 & $<0.001$ \\
\hline $\mathrm{T} 2$ & 284 & $21(24.1)$ & $94(26.3)$ & $104(36.6)$ & $65(42.5)$ & & & \\
\hline $\mathrm{T} 3$ & 86 & $\mathrm{I}(\mathrm{I} . \mathrm{I})$ & $4(1.1)$ & $27(9.5)$ & $54(35.3)$ & & & \\
\hline \multicolumn{9}{|l|}{ Grade } \\
\hline GI & 66 & $8(9.2)$ & 31 (8.7) & $21(7.4)$ & $6(3.9)$ & 0.026 & 0.217 & 0.115 \\
\hline G2 & $47 I$ & $42(48.3)$ & $208(58.3)$ & 149 (52.5) & $72(47.1)$ & & & \\
\hline G3-4 & 344 & $37(42.5)$ & II 8 (33.0) & II 4 (40.I) & $75(49.0)$ & & & \\
\hline \multicolumn{9}{|l|}{ Nodal stage } \\
\hline No & 418 & $60(69.0)$ & $206(57.7)$ & $142(50.0)$ & $10(6.5)$ & $<0.001$ & 0.118 & $<0.001$ \\
\hline NI & 280 & I8 (20.7) & 118 (33.0) & $93(32.7)$ & $51(33.3)$ & & & \\
\hline N2 & 107 & $4(4.6)$ & $20(5.6)$ & 32 (II.3) & $52(34.0)$ & & & \\
\hline N3 & 75 & $5(5.7)$ & $13(3.6)$ & $17(6.0)$ & $40(26.1)$ & & & \\
\hline \multicolumn{9}{|l|}{ ER status } \\
\hline Negative & 88 & II (I2.6) & $23(6.4)$ & $36(12.7)$ & I8 (II.8) & 0.037 & 0.051 & 0.782 \\
\hline Positive & 793 & $76(87.4)$ & 334 (93.6) & $248(87.3)$ & I 35 (88.2) & & & \\
\hline \multicolumn{9}{|l|}{ PR status } \\
\hline Negative & 203 & $25(28.7)$ & $68(19.0)$ & 67 (23.6) & $43(28.1)$ & 0.070 & 0.046 & 0.300 \\
\hline Positive & 678 & 62 (7I.3) & $289(81.0)$ & $217(76.4)$ & 110 (7I.9) & & & \\
\hline
\end{tabular}

Notes: aBCS vs. BCS + RT vs. MAST vs. MAST + RT. ${ }^{\circ} B C S$ vs. BCS + RT. cMAST vs. MAST + RT.

Abbreviations: $B C S$, breast-conserving surgery; ER, estrogen receptor; GI, well differentiated; G2, moderately differentiated; G3, poorly differentiated; G4, undifferentiated; MAST, mastectomy; N, node; PR, progesterone receptor; RT, radiotherapy; $\mathrm{T}$, tumor.

On multivariate analyses, tumor size, histological grade, and ER status were found to be independent prognostic factors for BCSS and OS (Table 3). The types of surgical procedures were not an independent prognostic factor for survival outcomes. Patients who had undergone MAST had similar BCSS and OS rates in the multivariate analyses, regardless of whether PORT was performed. In addition, patients who received BCS plus PORT did not show improved BCSS and OS compared to patients who received BCS alone.

\section{Clinical value of PORT}

We further classified patients into the following subgroups to determine the clinical value of PORT in IMPC: low-risk group ( $\mathrm{T} 1-2 \mathrm{~N} 0 ; \mathrm{n}=410)$, intermediate-risk group $(\mathrm{T} 1-2 \mathrm{~N} 1$; $\mathrm{n}=266$ ), and high-risk group (T3N0 and T1-3N2-3; $\mathrm{n}=$ 205). Our results indicated that in patients who had undergone BCS, PORT did not improve BCSS (log-rank test: low-risk group, $p=0.072$; intermediate-risk group, $p=0.764$; highrisk group, $p=0.564$ ) or OS (log-rank test: low-risk group, $p=0.402$; intermediate-risk group, $p=0.734$; high-risk group, $p=0.413)$. Similarly, in patients who had undergone
MAST, PORT was not associated with better survival in the low-risk group (log-rank test: BCSS, $p=0.537$; OS, $p=0.394$ ), intermediate-risk group (log-rank test: BCSS, $p=0.981$; OS, $p=0.896$ ), or high-risk group (log-rank test: BCSS, $p=0.127$; OS, $p=0.069$ ).

\section{Discussion}

Owing to the low incidence of IMPC and the small sample sizes of previous studies, the clinical value of PORT for IMPC of the breast remains unclear. In this study, we used population-based analyses to examine the role of PORT in patients with IMPC and found that PORT did not improve the survival of patients in the overall cohort or in different subgroups based on recurrence risk, regardless of the types of surgical procedures.

LRR is a major factor in determining the use of PORT in breast cancer. Yu et $\mathrm{a}^{29}$ found that regardless of adjuvant PORT, IMPC patients had a significantly higher 5-year LRR rate than IDC patients $(20.9 \%$ vs. $6.7 \%, p=0.0024)$. A study by Chen and Ding 9 included 95 patients with IMPC, of whom 74 patients underwent MAST and 21 underwent BCS; PORT 
Table 2 Univariate prognostic analyses

\begin{tabular}{|c|c|c|c|c|}
\hline \multirow[t]{2}{*}{ Characteristics } & \multicolumn{2}{|l|}{ BCSS } & \multicolumn{2}{|l|}{ OS } \\
\hline & HR (95\% Cl) & $p$ & HR (95\% Cl) & $p$ \\
\hline \multicolumn{5}{|l|}{ Age (years) } \\
\hline$\leq 49$ & 1 & & I & \\
\hline $50-69$ & $1.347(0.533-3.399)$ & 0.529 & $1.386(0.654-2.940)$ & 0.395 \\
\hline$\geq 70$ & $1.258(0.422-3.75 \mathrm{I})$ & $0.68 \mathrm{I}$ & $3.254(1.528-6.930)$ & 0.002 \\
\hline \multicolumn{5}{|l|}{ Ethnicity } \\
\hline White & I & & 1 & \\
\hline Black & $1.967(0.795-4.864)$ & 0.143 & $1.640(0.852-3.158)$ & 0.139 \\
\hline Other & $0.933(0.279-3.123)$ & 0.911 & $0.55 \mid(0.199-1.528)$ & 0.252 \\
\hline \multicolumn{5}{|l|}{ Tumor size } \\
\hline TI & I & & 1 & \\
\hline $\mathrm{T} 2$ & $2.474(\mathrm{I} .023-5.98 \mathrm{I})$ & 0.044 & $1.973(1.138-3.420)$ & 0.016 \\
\hline T3 & $9.074(3.748-21.966)$ & $<0.001$ & $3.823(1.961-7.454)$ & $<0.001$ \\
\hline \multicolumn{5}{|l|}{ Grade } \\
\hline GI-2 & I & & I & \\
\hline G3-4 & $4.282(1.915-9.572)$ & $<0.001$ & $1.909(1.165-3.129)$ & 0.010 \\
\hline \multicolumn{5}{|l|}{ Nodal stage } \\
\hline No & 1 & & I & \\
\hline NI & $0.971(0.370-2.552)$ & 0.953 & I.0II (0.559-I.827) & 0.971 \\
\hline N2 & $2.473(0.898-6.809)$ & 0.08 & $1.615(0.779-3.350)$ & 0.198 \\
\hline N3 & $4.839(1.908-12.270)$ & 0.001 & $2.144(1.004-4.577)$ & 0.049 \\
\hline \multicolumn{5}{|l|}{ ER status } \\
\hline Negative & 1 & & 1 & \\
\hline Positive & $0.234(0.1$ I $2-0.488)$ & $<0.001$ & $0.392(0.223-0.691)$ & 0.001 \\
\hline \multicolumn{5}{|l|}{ PR status } \\
\hline Negative & 1 & & I & \\
\hline Positive & $0.434(0.213-0.887)$ & 0.022 & $0.567(0.340-0.945)$ & 0.030 \\
\hline \multicolumn{5}{|l|}{ Treatment } \\
\hline $\mathrm{BCS}+\mathrm{RT}$ & 1 & & I & \\
\hline $\mathrm{BCS}$ & $3.115(0.697-13.921)$ & 0.137 & $1.946(0.840-4.509)$ & 0.121 \\
\hline MAST & $4.962(1.659-14.847)$ & 0.004 & $2.106(1.157-3.833)$ & 0.015 \\
\hline MAST + RT & $4.762(1.434-15.818)$ & 0.011 & $\mathrm{I} .443(0.66 \mathrm{I}-3.153)$ & 0.357 \\
\hline
\end{tabular}

Abbreviations: BCS, breast-conserving surgery; BCSS, breast cancer-specific survival; Cl, confidence interval; ER, estrogen receptor; GI, well differentiated; G2, moderately differentiated; G3, poorly differentiated; G4, undifferentiated; HR, hazard ratio; MAST, mastectomy; N, node; OS, overall survival; PR, progesterone receptor; $\mathrm{RT}$, radiotherapy; $\mathrm{T}$, tumor.

was performed depending on the indications in individual patients. The 5-year LRR rates in the entire cohort and in nodepositive patients were $28.6 \%$ and $35.8 \%$, respectively, which were significantly higher than the rates in patients with triplenegative breast cancer (10.2\% and $18.3 \%$, respectively). The 5 -year LRR rates in patients with N0-, N1-, N2-, and N3-stage diseases were $\sim 10 \%, 20 \%, 40 \%$, and $50 \%$, respectively, ${ }^{9}$ which were significantly higher than the rates reported for IDC in prospective studies. ${ }^{27,28}$ As the N2 and N3 stages are absolute indications for PORT, the LRR rate was high in the study by Chen and Ding. ${ }^{9}$ However, a retrospective multicenter casecontrol study from Korea found that regardless of PORT, the LRR rate was significantly higher in IMPC than in IDC (8.2\% vs. $3.7 \%, p=0.03) .{ }^{10}$ Other studies have not found differences in LRR between the IMPC and IDC subtypes..$^{24,31}$ Thus, the reported LRR rates of IMPC vary widely, and this may have an impact on the use of PORT. Owing to the limitations of the SEER database, we were unable to obtain the LRR data of the patients. Although more than half of the patients had lymph node metastases, the 5-year BCSS was $95.7 \%$ in our study, which is similar to that reported in the two previous SEER studies. ${ }^{25,26}$ These results suggest that IMPC patients have favorable survival outcomes, which are not inferior to those of IDC patients. ${ }^{25,26}$

To date, no study has specifically aimed to determine the value of PORT in patients with IMPC. In a retrospective multicenter case-control study from Korea, of the 2, 17, and 75 patients with N0-, N1-, and N2-3-stage diseases who underwent breast/chest wall radiotherapy with or without regional lymph node radiotherapy, a total of 0,1 , and 12 patients developed LRR, which was significantly higher than the LRR rates in patients with IDC $(p=0.03)$. However, PORT was not associated with reduced LRR in IMPC $(p=0.94) .{ }^{10} \mathrm{Yu}$ et al ${ }^{29}$ included 72 IMPC patients, of whom 
Table 3 Multivariate prognostic analyses

\begin{tabular}{|c|c|c|c|c|}
\hline \multirow[t]{2}{*}{ Characteristics } & \multicolumn{2}{|l|}{ BCSS } & \multicolumn{2}{|l|}{ OS } \\
\hline & HR (95\% Cl) & $p$ & HR (95\% CI) & $p$ \\
\hline \multicolumn{5}{|l|}{ Age (years) } \\
\hline$\leq 49$ & - & & I & \\
\hline $50-69$ & - & - & $1.416(0.664-3.020)$ & 0.368 \\
\hline$\geq 70$ & - & - & $4.837(2.215-10.564)$ & $<0.001$ \\
\hline \multicolumn{5}{|l|}{ Tumor size } \\
\hline TI & I & & I & \\
\hline $\mathrm{T} 2$ & $2.126(0.875-5.163)$ & 0.096 & $2.090(I .174-3.72 I)$ & 0.012 \\
\hline $\mathrm{T} 3$ & $7.756(3.195-18.827)$ & $<0.001$ & $5.229(2.4 \mid 4-11.325)$ & $<0.001$ \\
\hline \multicolumn{5}{|l|}{ Grade } \\
\hline GI-2 & 1 & & I & \\
\hline G3-4 & $3.270(1.437-7.440)$ & 0.005 & $1.720(1.026-2.886)$ & 0.040 \\
\hline \multicolumn{5}{|l|}{ Nodal stage } \\
\hline No & I & & I & \\
\hline $\mathrm{NI}$ & $0.799(0.295-2.166)$ & 0.659 & 1.231 (0.660-2.297) & 0.514 \\
\hline N2 & $0.986(0.297-3.280)$ & 0.982 & $1.190(0.498-2.844)$ & 0.695 \\
\hline N3 & $1.623(0.493-5.344)$ & 0.426 & $1.816(0.735-4.484)$ & 0.196 \\
\hline \multicolumn{5}{|l|}{ ER status } \\
\hline Negative & 1 & & I & \\
\hline Positive & $0.339(0.160-0.720)$ & 0.005 & $0.391(0.215-0.709)$ & 0.002 \\
\hline \multicolumn{5}{|l|}{ PR status } \\
\hline Negative & I & & I & \\
\hline Positive & I.483 (0.442-4.976) & 0.523 & $0.945(0.437-2.046)$ & 0.886 \\
\hline \multicolumn{5}{|l|}{ Treatment } \\
\hline $\mathrm{BCS}+\mathrm{RT}$ & 1 & & I & \\
\hline BCS & $2.42 I(0.527-11.124)$ & 0.256 & $2.122(0.903-4.986)$ & 0.084 \\
\hline MAST & $2.528(0.80 \mathrm{I}-7.980)$ & 0.114 & I.63I (0.864-3.078) & 0.132 \\
\hline MAST + RT & $1.334(0.342-5.210)$ & 0.678 & $0.762(0.316-1.836)$ & 0.546 \\
\hline
\end{tabular}

Note: '-' indicates no data.

Abbreviations: BCS, breast-conserving surgery; BCSS, breast cancer-specific survival; Cl, confidence interval; ER, estrogen receptor; GI, well differentiated; G2, moderately differentiated; G3, poorly differentiated; G4, undifferentiated; HR, hazard ratio; MAST, mastectomy; N, node; OS, overall survival; PR, progesterone receptor; $\mathrm{RT}$, radiotherapy; $\mathrm{T}$, tumor.

$71.3 \%$ received PORT; the 5-year LRR rate was $20.9 \%$ in the entire cohort. A study from China included 100 patients with IMPC, of whom $72 \%$ received PORT, and $11.2 \%$ of the entire cohort developed LRR. ${ }^{32}$ Li et al ${ }^{24}$ included 33 patients with IMPC, all of whom underwent MAST; 78.2\% were lymph node positive, and $42.4 \%$ of all patients received PORT, but only one patient developed LRR. Vingiani et a ${ }^{31}$ included 49 patients with IMPC, of whom $87.8 \%$ received PORT; the LRR rate was $6.1 \%$. We were unable to obtain the LRR data of patients who had undergone PORT in the abovementioned three studies. A SEER study by Chen et $\mathrm{al}^{25}$ found that PORT was an independent predictor of disease-specific survival and OS in IMPC. However, the impact of PORT after specific types of surgical procedures and in specific risk factor-based groups was not further analyzed.

In the present study, univariate analysis showed similar survival in patients who underwent BCS alone and in those who underwent BCS plus PORT; patients who underwent MAST with or without PORT had poorer survival than those who underwent BCS plus PORT. Selection bias might account for this finding, as patients with adverse prognostic factors, such as younger age, large tumors, and advanced nodal stage, were more likely to undergo MAST with or without PORT. After adjustments for age, tumor size, tumor grade, nodal stage, ER status, and PR status in the multivariate analyses, patients who underwent BCS alone and those who underwent MAST with or without PORT had similar BCSS and OS to those who underwent BCS plus PORT. Studies on IDC have demonstrated that the improved local control rate in PORT groups is sequentially translated to improvement in the survival rate of patients with distant metastases. ${ }^{27,28}$ However, the BCSS and OS rates were similar in the PORT and non-PORT groups in our study, regardless of the type of surgical procedures. In addition, PORT did not improve the survival of patients after BCS or MAST in the low-risk, intermediate-risk, and high-risk groups.

The reasons why PORT did not improve survival in patients after BCS or in high-risk patients after MAST remain unknown. Possible explanations are as follows: 1) we could not obtain the patterns of LRR in the SEER database. 
However, IMPC is associated with several risk factors for LRR, including LVI and high probability of lymph node metastasis. In randomized studies of breast cancer, especially for patients who have undergone BCS, individual studies often find significant differences in local recurrence, but not in OS. ${ }^{33,34}$ Survival changes due to addition of PORT are often only seen with larger meta-analyses; ${ }^{35,36}$ 2) although IMPC includes several high risk factors for LRR, the risk of distant metastasis is not higher in IMPC compared to IDC, 3,10,24,29,32 which suggests that IMPC has a special biological behavior; and 3 ) the vast majority of IMPC patients in this study had ER-positive disease and may have benefited from endocrine therapy, which may have reduced the potential risk of LRR.

Similar to a previous SEER study, ${ }^{25}$ approximately half of the patients in our study underwent BCS, and the BCSS and OS rates in the BCS and MAST groups were similar. Studies have shown that MAST is the most common type of surgical procedure in IMPC and that the types of surgical procedures were not associated with survival outcomes. ${ }^{9,24,37}$ However, in a retrospective multicenter case-control study from Korea $(n=267), 57.7 \%$ of patients underwent BCS, and BCS patients were found to have better LRR control rates than MAST patients $(96.7 \%$ vs. $86.5 \%, p=0.03) .{ }^{10}$ Therefore, the survival outcomes of BCS were not inferior to those of MAST in patients with IMPC of the breast.

We need to acknowledge several limitations of our study. First, retrospective studies have inherent bias. Second, detailed histopathological data, including LVI status and resection margin status, were unavailable in the SEER database. Data on the use of systemic therapies (i.e., chemotherapy or endocrine therapy) were also lacking in the SEER database. In addition, the target volume and radiation dose were not recorded, and the patterns of LRR in patients with and without PORT were also unknown. Finally, it has been shown that there are many inaccuracies in the SEER databases, with high rates of underreporting for PORT receipt. ${ }^{38}$ However, the primary strength of this study is that we investigated the role of PORT in patients with IMPC of the breast, by using a large population-based database. Therefore, this study population is more diversified and potentially more generalizable than retrospective studies from single institutions.

\section{Conclusion}

IMPC has favorable BCSS and OS rates. Regardless of the types of surgical procedures (MAST or BCS), PORT groups were not inferior to non-PORT groups on BCSS and OS in
IMPC patients. Further prospective large-scale studies are necessary to confirm the clinical value of PORT in IMPC.

\section{Acknowledgments}

This work was partly supported by the Natural Science Foundation of Fujian Province (No. 2016J01635), Science and Technology Planning Projects of Xiamen Science \& Technology Bureau (No. 3502Z20174070), and Guangdong Medical Research Foundation (No. A2017023).

\section{Disclosure}

The authors report no conflicts of interest in this work.

\section{References}

1. Tavassoli FA, Devilee P. Pathology and Genetics of Tumours of the Breast and Female Genital Organs. World Health Organization Classification of Tumours. Lyon: IARC Press; 2003:35-36.

2. Siriaunkgul S, Tavassoli FA. Invasive micropapillary carcinoma of the breast. Mod Pathol. 1993;6(6):660-662.

3. Zekioglu O, Erhan Y, Ciris M, Bayramoglu H, Ozdemir N. Invasive micropapillary carcinoma of the breast: high incidence of lymph node metastasis with extranodal extension and its immunohistochemical profile compared with invasive ductal carcinoma. Histopathology. 2004;44(1):18-23.

4. Luna-Moré S, de los Santos F, Bretón JJ, Cañadas MA. Estrogen and progesterone receptors, c-erbB-2, p53, and Bcl-2 in thirty-three invasive micropapillary breast carcinomas. Pathol Res Pract. 1996;192(1):27-32.

5. Kuroda H, Sakamoto G, Ohnisi K, Itoyama S. Clinical and pathologic features of invasive micropapillary carcinoma. Breast Cancer. 2004; 11(2):169-174.

6. De la Cruz C, Moriya T, Endoh M, et al. Invasive micropapillary carcinoma of the breast: clinicopathological and immunohistochemical study. Pathol Int. 2004;54(2):90-96.

7. Pettinato G, Manivel CJ, Panico L, Sparano L, Petrella G. Invasive micropapillary carcinoma of the breast: clinicopathologic study of 62 cases of a poorly recognized variant with highly aggressive behavior. Am J Clin Pathol. 2004;121(6):857-866.

8. Guo XJ, Chen L, Lang RG, Fan Y, Fu L. [Relationship between lymph node metastasis and pathologic features of invasive micropapillary carcinoma of breast] . Zhonghua Bing Li Xue Za Zhi. 2006;35(1):8-12. Chinese.

9. Chen HL, Ding A. Comparison of invasive micropapillary and triple negative invasive ductal carcinoma of the breast. Breast. 2015;24(6): 723-731.

10. Yu JI, Choi DH, Huh SJ, et al. Differences in prognostic factors and failure patterns between invasive micropapillary carcinoma and carcinoma with micropapillary component versus invasive ductal carcinoma of the breast: retrospective multicenter case-control study (KROG 13-06). Clin Breast Cancer. 2015;15(5):353-361.e1-2.

11. Acs G, Esposito NN, Rakosy Z, Laronga C, Zhang PJ. Invasive ductal carcinomas of the breast showing partial reversed cell polarity are associated with lymphatic tumor spread and may represent part of a spectrum of invasive micropapillary carcinoma. Am J Surg Pathol. 2010;34(11):1637-1646.

12. Nagi C, Guttman M, Jaffer S, et al. N-cadherin expression in breast cancer: correlation with an aggressive histologic variant - invasive micropapillary carcinoma. Breast Cancer Res Treat. 2005;94(3):225-235.

13. Lim MG, Adsay NV, Grignon DJ, Osunkoya AO. E-cadherin expression in plasmacytoid, signet ring cell and micropapillary variants of urothelial carcinoma: comparison with usual-type high-grade urothelial carcinoma. Mod Pathol. 2011;24(2):241-247. 
14. Kuba S, Ohtani H, Yamaguchi J, et al. Incomplete inside-out growth pattern in invasive breast carcinoma: association with lymph vessel invasion and recurrence-free survival. Virchows Arch . 2011;458(2):159-169.

15. Li YS, Kaneko M, Sakamoto DG, Takeshima Y, Inai K. The reversed apical pattern of MUC1 expression is characteristics of invasive micropapillary carcinoma of the breast. Breast Cancer. 2006;13(1): 58-63.

16. Gong Y, Sun X, Huo L, Wiley EL, Rao MS. Expression of cell adhesion molecules, CD44s and E-cadherin, and microvessel density in invasive micropapillary carcinoma of the breast. Histopathology. 2005;46(1):24-30.

17. Li W, Liu F, Lei T, et al. The clinicopathological significance of CD44+/ CD24-/low and CD24+ tumor cells in invasive micropapillary carcinoma of the breast. Pathol Res Pract. 2010;206(12):828-834.

18. Moritani S, Ichihara S, Hasegawa M, et al. Serous papillary adenocarcinoma of the female genital organs and invasive micropapillary carcinoma of the breast. Are WT1, CA125, and GCDFP-15 useful in differential diagnosis? Hum Pathol. 2008;39(5):666-671.

19. Wei J, Cui L, Liu F, et al. E-selectin and Sialyl Lewis X expression is associated with lymph node metastasis of invasive micropapillary carcinoma of the breast. Int J Surg Pathol. 2010;18(3):193-200.

20. Liu F, Lang R, Wei J, et al. Increased expression of SDF-1/CXCR4 is associated with lymph node metastasis of invasive micropapillary carcinoma of the breast. Histopathology. 2009;54(6):741-750.

21. Cui LF, Guo XJ, Wei J, et al. Overexpression of TNF-alpha and TNFRII in invasive micropapillary carcinoma of the breast: clinicopathological correlations. Histopathology. 2008;53(4):381-388.

22. Nassar H, Wallis T, Andea A, Dey J, Adsay V, Visscher D. Clinicopathologic analysis of invasive micropapillary differentiation in breast carcinoma. Mod Pathol. 2001;14(9):836-841.

23. Paterakos M, Watkin WG, Edgerton SM, Moore DH 2nd, Thor AD. Invasive micropapillary carcinoma of the breast: a prognostic study. Hum Pathol. 1999;30(12):1459-1463.

24. Li G, Yang S, Yao J, et al. Invasive micropapillary carcinoma of the breast had poor clinical characteristics but showed no difference in prognosis compared with invasive ductal carcinoma. World J Surg Oncol. 2016;14(1):207.

25. Chen AC, Paulino AC, Schwartz MR, et al. Population-based comparison of prognostic factors in invasive micropapillary and invasive ductal carcinoma of the breast. Br J Cancer. 2014;111(3):619-622.

26. Chen AC, Paulino AC, Schwartz MR, et al. Prognostic markers for invasive micropapillary carcinoma of the breast: a population-based analysis. Clin Breast Cancer. 2013;13(2):133-139.

27. Fisher B, Anderson S, Bryant J, et al. Twenty-year follow-up of a randomized trial comparing total mastectomy, lumpectomy, and lumpectomy plus irradiation for the treatment of invasive breast cancer. $N E n g l$ J Med. 2002;347(16):1233-1241.
28. Overgaard M, Hansen PS, Overgaard J, et al. Postoperative radiotherapy in high-risk premenopausal women with breast cancer who receive adjuvant chemotherapy. Danish Breast Cancer Cooperative Group 82b Trial. N Engl J Med. 1997;337(14):949-955.

29. Yu JI, Choi DH, Park W, et al. Differences in prognostic factors and patterns of failure between invasive micropapillary carcinoma and invasive ductal carcinoma of the breast: matched case-control study. Breast. 2010;19(3):231-237.

30. Surveillance, Epidemiology, and End Results (SEER) Program [homepage on the Internet]. SEER*Stat Database: Incidence - SEER 18 Regs Research Data + Hurricane Katrina Impacted Louisiana Cases, Nov 2016 Sub (1973-2014 varying) - Linked To County Attributes - Total U.S., 1969-2015 Counties, National Cancer Institute, DCCPS, Surveillance Research Program, Surveillance Systems Branch, released April 2017, based on the November 2016 submission. Available from: https:// seer.cancer.gov/data/. Accessed March 4, 2017.

31. Vingiani A, Maisonneuve P, Dell'orto P, et al. The clinical relevance of micropapillary carcinoma of the breast: a case-control study. Histopathology. 2013;63(2):217-224.

32. Chen L, Fan Y, Lang RG, et al. Breast carcinoma with micropapillary features: clinicopathologic study and long-term follow-up of 100 cases. Int J Surg Pathol. 2008;16(2):155-163.

33. Killander F, Karlsson P, Anderson H, et al. No breast cancer subgroup can be spared postoperative radiotherapy after breast-conserving surgery. Fifteen-year results from the Swedish Breast Cancer Group randomised trial, SweBCG 91 RT. Eur J Cancer. 2016;67:57-65.

34. Early Breast Cancer Trialists' Collaborative Group. Favourable and unfavourable effects on long-term survival of radiotherapy for early breast cancer: an overview of the randomised trials. Lancet. 2000;355(9217):1757-1770.

35. EBCTCG (Early Breast Cancer Trialists' Collaborative Group), McGale $\mathrm{P}$, Taylor C, et al. Effect of radiotherapy after mastectomy and axillary surgery on 10-year recurrence and 20-year breast cancer mortality: meta-analysis of individual patient data for 8135 women in 22 randomised trials. Lancet. 2014;383(9935):2127-2135.

36. Early Breast Cancer Trialists' Collaborative Group (EBCTCG), Darby S, McGale P, et al. Effect of radiotherapy after breast-conserving surgery on 10-year recurrence and 15-year breast cancer death: meta-analysis of individual patient data for 10,801 women in 17 randomised trials. Lancet. 2011;378(9804):1707-1716.

37. Liu Y, Huang X, Bi R, Yang W, Shao Z. Similar prognoses for invasive micropapillary breast carcinoma and pure invasive ductal carcinoma: a retrospectively matched cohort study in China. PLoS One. 2014;9(9):e106564.

38. Jagsi R, Abrahamse P, Hawley ST, Graff JJ, Hamilton AS, Katz SJ. Underascertainment of radiotherapy receipt in surveillance, epidemiology, and end results registry data. Cancer. 2012;118(2):333-341.
Cancer Management and Research

\section{Publish your work in this journal}

Cancer Management and Research is an international, peer-reviewed open access journal focusing on cancer research and the optimal use of preventative and integrated treatment interventions to achieve improved outcomes, enhanced survival and quality of life for the cancer patient. The manuscript management system is completely online and includes

\section{Dovepress}

a very quick and fair peer-review system, which is all easy to use. Visit http://www.dovepress.com/testimonials.php to read real quotes from published authors. 